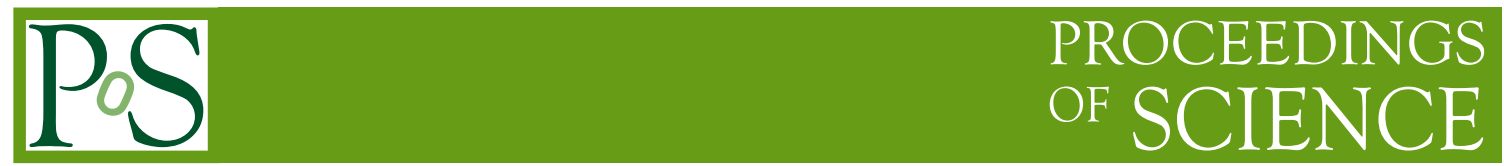

\title{
Measurements of Top Quark Properties at the LHC
}

\author{
Hongbo Liao*† \\ E-mail: hongbo.liao@cern.ch
}

\begin{abstract}
A summary on the most recent results of the ATLAS and CMS Collaborations on the top quark porperties is presented. The measurements used data from the 2010 and 2011 LHC run, using integrated luminosities ranging from $35 \mathrm{pb}^{-1}$ to $5.0 \mathrm{fb}^{-1}$, the latter corresponding to the full 2011 data sample.
\end{abstract}

The XIth International Conference on Heavy Quarks and Leptons,

June 11-15, 2012

Prague, Czech Republic

\footnotetext{
*Speaker.

${ }^{\dagger}$ On behalf of the ATLAS and CMS Collaborations
} 

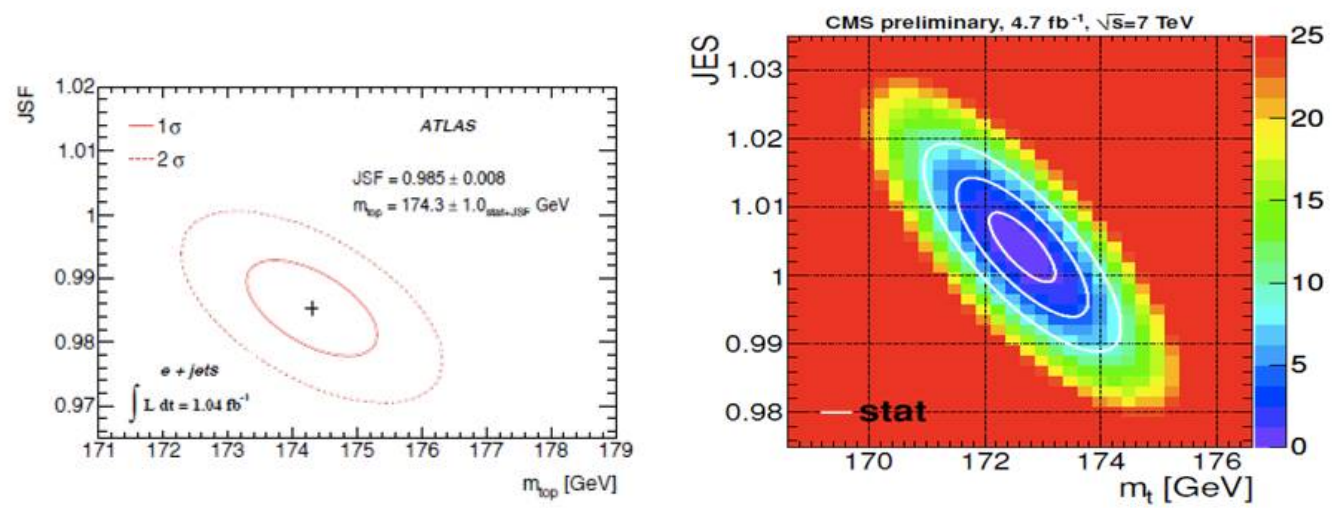

Figure 1: Left: The ATLAS results of the 2d-analysis when performed on data for the $e+$ jets channel. The ellipese correspond to $1 \sigma$ and $2 \sigma$ uncertainties of $m_{t}$ and JSF. Right : The CMS results of the 2d likelihood measured. The controus crosspond to 1,2 and $3 \sigma$ uncertainty.

\section{Introduction}

The top quark completes the three generation of the Standard Model (SM). After QCD jets, $\mathrm{W}$ and $\mathrm{Z}$ bosons, the production of top quark is the dominant process in pp collisions at LHC. Therefore studying the top quark properties at the LHC is of great interest since it can lead to precise tests of the Standard Model (SM), predictions in terms of top quark mass, charge, the branching ratio, spin and the $W t b$ vertex. It also allow one to look for new physics, which is likely to show up in the top sector, by searching for rare decays, anomalous couplings, charge asymmetry, Flavour Changing Neutral Currents (FCNC). A short summary of the most recent results by the ATLAS and CMS Collaborations is presented here.

\section{Top Quark Mass}

\subsection{Measurement of the top-quark mass in the lepton+jets channel}

Both ATLAS and CMS have measured $m_{t}$ in the lepton+jets channel.

ATLAS is using two different approaches to measure $m_{t}$, designed to reduce the JES uncertainty [1]. Both analyses are based on the template method. In the 1d-analysis, $R_{32}=\frac{m_{t}^{\text {reco }}}{m_{W}^{\text {reco }}}$ is calculated for every event, where $m_{t}^{\text {reco }}$ and $m_{W}^{\text {reco }}$ are the reconstructed invariant masses of the hadronically decaying top quark and $\mathrm{W}$ boson, respectively. The $2 \mathrm{~d}$-analysis, a combined measurement of $m_{t}$ and a global Jet energy Scale Factor (JSF), is performed by a template fit to $m_{t}^{\text {reco }}$ and $m_{W}^{\text {reco }}$. Both analyses are applied on $1.04 \mathrm{fb}^{-1}$ of data. The $2 \mathrm{~d}$-analysis has a slightly smaller uncertainty: $m_{t}=174.5 \pm 0.6$ (stat) \pm 2.3 (syst) GeV. The left of Figures 1 shows the ATLAS results of the $2 \mathrm{~d}$-analysis when performed on data for the $e+$ jets channel. The ellipese correspond to $1 \sigma$ and $2 \sigma$ uncertainties of $m_{t}$ and JSF. The systematic uncertainty is dominated by the uncertainty on ISR/FSR and the uncertainty on the b-jet energy scale.

The CMS measurement of $m_{t}$ uses $4.7 \mathrm{fb}^{-1}$ of data in the $\mu+$ jets channel [2]. To extract $m_{t}$ from the data, the Ideogram method is used in a combined measurement of $m_{t}$ and the Jet Energy 
Scale (JES). for all possible jet-quark assignments (correct as- signments, wrong assignments and unmatched assignments) are taken from simulation. The individual event likelihoods are combined in a global likelihood, from which the measured $\mathrm{mt}$ and JES values can be extracted. With this method, CMS measures $m_{t}=172.6 \pm 0.6$ (stat) \pm 1.2 (syst) GeV. The right of Figures 1 shows the CMS results of the $2 \mathrm{~d}$ likelihood measured. The controus crosspond to 1, 2 and $3 \sigma$ uncertainty. The systematic uncertainty is dominated by the b-jet energy scale uncertainty and the uncertainty on the factorization scale. The systematic uncertainty does not include the uncertainty on color reconnection and on the underlying event.

\subsection{Measurement of the top-quark mass in the dilepton channel}

The $m_{t}$ measurement from CMS in the dilepton channel is performed with $2.3 \mathrm{fb}^{-1}$ [3].

The event-by-event top-quark mass $m_{K I N b}$ is reconstructed with the KINb algorithm. For each of the possible jet-quark assignments the kinematic equations are solved multiple times per event, each time varying the reconstructed kinematics within their resolutions. The jet-quark assignment with the largest number of solutions is selected. Finally, $m_{K I N b}$ is extracted by taking the mean of a Gaussian to the distribution of the reconstructed top quark mass for all the different solutions of the kinematic equations, for the chosen jet-quark assignment. The extraction of $m_{t}$ is then performed by applying the template method. Templates which are sensitive to $m_{t}$ are constructed for different top-quark mass hypotheses. The value of $m_{t}$ is then extracted by doing a maximum likelihood fit of these templates to the distribu- tion of $m_{K I N b}$ observed in data. By applying this technique, CMS measures $m_{t}=173.3 \pm 1.2$ (stat) \pm 2.5 (syst) $\mathrm{GeV}$, where the systematic uncertainty is dominated by the global Jet Energy Scale (JES) uncertainty and the uncertainty on the favour-dependent JES.

\subsection{Measurement of the top-quark mass in the all-jets channel}

ATLAS performed the first measurement at the LHC of $m_{t}$ in the all-jets channel, using 2.04 $f b^{-1}$ of data [4].

The $t \bar{t}$ event topology is reconstructed using a mass $\chi^{2}$ For every event, the jet-quark assignment with the lowest $\chi^{2}$ is taken. This $\chi^{2}$ is minimized as a function of $m_{W}$ and $m_{t}$. Only events with $\chi^{2}<8$ are considered in the extraction of $\mathrm{mt}$. The template method is applied to the distribution of $m_{j j b}$ values from the selected jet-quark assignment, as observed in data. ATLAS measures $m_{t}=174.9 \pm 2.1$ (stat) \pm 3.8 (syst) $\mathrm{GeV}$. The systematic uncertainty is dominated by the uncertainty on Initial and Final State Radiation (ISR/FSR), the uncertainty on the data-driven multijet background and the JES uncertainty.

\subsection{Measurement of the top-quark mass from the $t \bar{t}$ cross section at $\sqrt{s}=7 \mathrm{TeV}$}

One of the problems of the direct measurements of $m_{t}$ is that they use the mass definition from Monte Carlo generators, which is not related to $\mathrm{mt}$ in a well-defined renormalization scheme $\left(m_{t}^{\text {pole }}\right.$ or $m_{t}^{M S}$ ) in a straight forward way. These masses can however be extracted from the measured cross section of top quark pair production, since the theoretical dependency of on $m_{t}^{\text {pole }}$ or $m_{t}^{M S}$ is known. ATLAS used measured from $35 \mathrm{pb}^{-1}$ of data in the lepton+jets channel, and obtains $m_{t}^{\text {pole }}$ $=166.4_{-7.3}^{+7.8} \mathrm{GeV}$ for the pole mass [5]. CMS used measured in the dilepton final state from 1.1 $f b^{-1}$ of data, and measures $m_{t}^{\text {pole }}=170.3_{-6.7}^{+7.3} \mathrm{GeV}[6]$. Both the ATLAS and CMS results are in agreement with previous measurement performed by CDF and D0. 


\subsection{Measurement of the mass difference between top and antitop quarks}

One of the fundamental symmetries in the standard model, the invariance under CPT transformations, can be tested by measuring the difference in mass between a particle and the corre- sponding antiparticle. Since the top quark is the only quark that decays before hadronization can take place, this difference can be measured directly. CMS performed a measurement using $5.0 \mathrm{fb}^{-1}$ of data in the lepton+jets channel [7]. The events were splitted in two distinct samples according to the charge of the lepton. In each of these two samples, the mass of the hadronically decaying top quarks was measured and finally both masses were subtracted from each other. This resulted in $\Delta m_{t}=-0.44 \pm 0.46$ (stat) \pm 0.27 (syst) GeV. The smallness of the systematic uncertainty, when compared to $m_{t}$ measurements, can be explained by the cancellation of most systematics by taking the difference.

\section{Top Quark Charge}

Both the CMS [8] and ATLAS [9] Collaborations have excluded at more than $5 \sigma$ the top quark having a charge $-4 / 3 \mathrm{e}$, in favour of the standard model (SM) prediction of $2 / 3 \mathrm{e}$. The charge of the $\mathrm{W}$ is identified through the charge of the lepton; the charge of the b-jet is determined from the presence of a soft lepton inside the jet.

\section{Top Branching Ratios}

In the SM, the top is expected to decay $100 \%$ of the time to a W- boson and a b-quark. Thus the observation of any other decay mode would be a sign of new physics. Using data in the dilepton channel and looking at the multiplicity of the identified heavy flavour jets, the CMS Collaboration was able to measure the branching ratio to $\mathrm{Wb}$ to a W-boson and any quark (Wq) to be $0.98 \pm 0.04$ and place the $95 \%$ confidence level around 0.85 [10].

\section{Flavour Changing Neutral Currents}

In addition to looking for anomalous decays to a W boson and a non-b-quark, one can look for flavour changing neutral current decay modes tqZ. Many models of new physics predict measurable branching ratios in this decay mode. Results from the ATLAS [11] and CMS [12] Collaborations have put upper limits on this fraction at $0.73 \%$ and $0.34 \%$, respectively. In these analyses, events with three leptons are considered, where two of the leptons should be compatible with a Z-boson and the other originate from the other top quark that decays to $\mathrm{Wb}$.

One can also search for flavour changing neutral currents, not in the decay mode, but in the production mode. The ATLAS Collaboration looked for production modes where a coupling between a gluon, a top quark and a light quark could exist [13]. These signatures would be similar to single top production but with different kinematics. In order to separate this new signature from the SM backgrounds, a neutral network is used. Stringent limits are placed on the maximum allowed branching ratios of top to ug and cg at the level of $5.710^{-5}$ and $2.710^{-4}$, respectively. 


\section{W boson polarisation}

In order the measure the three components of the polarisation of the W-boson, the distribution of the $\cos \theta^{\star}$ is studied. This angle represents the angle between the lepton momentum in the $\mathrm{W}$ rest-frame and the momentum of the $\mathrm{W}$ in the top rest-frame. After subtracting the background distributions, the observed $\cos \theta^{\star}$ distribution is corrected to particle-level. The resulting distribution is then fitted to templates of each of the three polarization components, with the constraint that the total fraction has to be unity. The SM predicts contribution close to zero for the righthanded polarisation, about $31 \%$ left-handed and $69 \%$ longitudinal polarisation. Both the ATLAS [14] and CMS [15] results are compatible with the SM predictions. The right handed polarisation component, FR, is predicted to be $0.0017 \pm 0.0001$ at Next to Next to Leading Order, and is measured to be $0.01 \pm 0.01$ (stat) \pm 0.04 (syst) and $0.040 \pm 0.035$ (stat) \pm 0.044 (syst) by the ATLAS and CMS Collaborations, respectively. These results are then used to place stringent limits on anomalous couplings, described by an effective field theory, given that the scale of new physics is larger than the observable region.

\section{Charge asymmetry}

Unlike the $p \bar{p}$ collisions from the Tevatron, the LHC pp collisions cannot give a direct forwardbackward asymmetry in the production of top quark pairs. Yet owing to the Parton Distribution Functions being different for quarks and anti-quarks, the difference in rapidity (y) distributions of the top and anti-top can be used to measure a form of asymmetry; indeed the top quark spectrum in $y$ is expected to be slightly broader than for that of the anti- top. By using the difference in absolute rapidity between the top and anti-top one can dene an asymmetry

$$
A_{C}=\frac{N(\Delta|y|>0)-N(\Delta|y|<0)}{N(\Delta|y|>0)+N(\Delta|y|<0)}
$$

Predictions from the MC@NLO generator are at the level of 0.006 \pm 0.002 . The measurement of a larger asymmetry would be an indication of new physics. Both the ATLAS [16] and CMS [17] Collaborations have studied this asymmetry in a variety of different kinematic regions. Both collaborations used the lepton+jets decay mode where one $\mathrm{W}$ decays leptonically and the other hadronically and both relied on the identification of at least one heavy flavour (b-tagged) jet. The measurements are corrected for detector and acceptance effects. The overall measurements of $A_{C}$ are compatible with zero. ATLAS measures an overall charge asymmetry of $A_{C}=$ $0.018 \pm 0.028$ (stat) \pm 0.023 (syst), while CMS measures a value of $A_{C}=0.004 \pm 0.010$ (stat) \pm 0.012 (syst). This asymmetry was also measured for different kinematic regions: for different values of the $t$ invariant mass (ATLAS and CMS) and as a function of the transverse momentum (pT) of the tt system (CMS). Respectively, the $A_{C}$ distribution as a function of the $t \bar{t}$ invariant mass from the ATLAS and CMS Collaborations are shown in the right and left of Figures 2. These results disfavour many of the models developed to account for the forward-backward asymmetry observed at the Tevatron.

The charge asymmetry from $t \bar{t}$ is transmitted to the leptons. It is possible to also measure a purely leptonic based asymmetry by looking at the rapidity difference between $l^{+}$and 

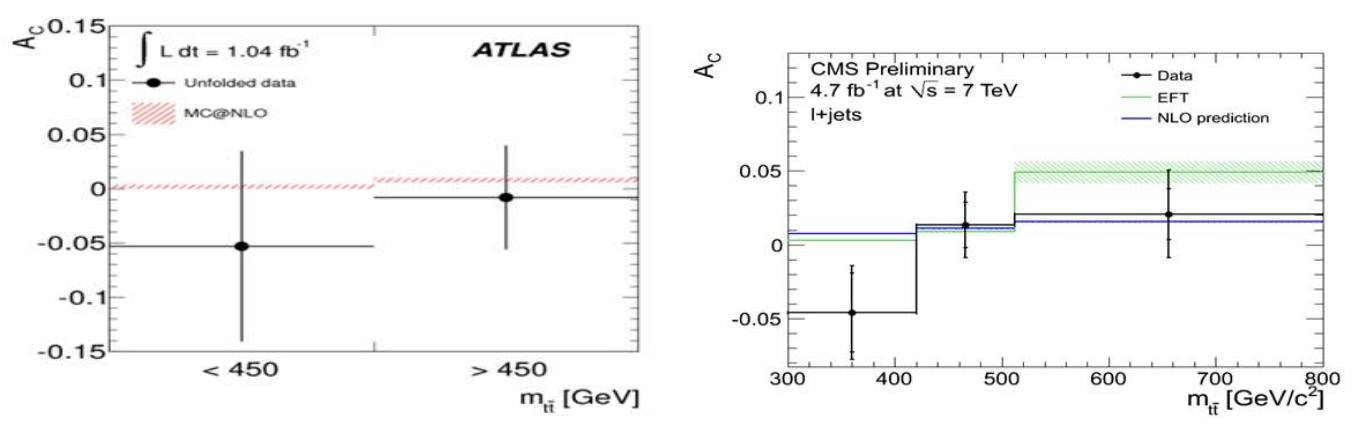

Figure 2: Left: The ATLAS results of the $A_{C}$ when $t \bar{t}$ invariant mass is in different region. Right : The $A_{C}$ distribution as a function of the $t \bar{t}$ invariant mass from the CMS.

$l^{-}$. SM predicts the level of $0.004 \pm 0.001$ for $A_{C}^{l l}$. ATLAS measures the leptonic based charge asymmetry of $A_{C}^{l l}=0.023 \pm 0.012$ (stat) \pm 0.008 (syst) and the overall charge asymmetry of $A_{C}^{t \bar{t}}=$ $0.057 \pm 0.024$ (stat) \pm 0.015 (syst) in the dilepton channel [18]. Combined ATLAS measurements in the dilepton channel and lepton+jets channel, give $A_{C}^{t \bar{t}}=0.029 \pm 0.018$ (stat) \pm 0.014 (syst). All of the measurements are consistent with the SM prediction.

\section{Spin Correlations}

The measurement of the spin correlation between the top and the anti-top quark is sensitive to the fact that the life-time of the top quark is so short that it decays before it hadronises and thus for $t \bar{t}$ events, one expects the spin structure to be preserved. In top decays where both W-bosons decay leptonically, the angular separation between the two leptons is sensitive to the presence of such correlations. The ATLAS Collaboration is able to exclude at more than $3 \sigma$ the scenario with no spin correlations, and measures a degree of spin correlation compatible with that predicted by the SM [19].

\section{Summary}

Top properties have been investigated by ATLAS and CMS Collaborations with the data collected in 2010 and 2011, correponding to an integrated luminosity from $35 \mathrm{pb}^{-1}$ to $5.0 \mathrm{fb}^{-1}$. There are already several LHC measurements worlds best now, such as the observation of spin correlations and the measurement of W polarization. So far no deviations from the SM expectation have been seen in those measurements.

Most analyses are not statistically limited and it is expected that with better understanding of the detector, the largest systematic effects will be much better under control. This will improve the analysis results.

\section{References}

[1] The ATLAS Collaboration, Submittes to EPJC (arXiv:1203.5755).

[2] The CMS Collaboration, CMS-PAS-TOP-11-015. 
[3] The CMS Collaboration, CMS-PAS-TOP-11-016.

[4] The ATLAS Collaboration, ATLAS-CONF-2011-030.

[5] The ATLAS Collaboration, ATLAS-CONF-2011-054.

[6] The CMS Collaboration, CMS-PAS-TOP-11-008.

[7] The CMS Collaboration, Submittes to JHEP (arXiv:1204.2807).

[8] The CMS Collaboration, CMS-PAS-TOP-11-031.

[9] The ATLAS Collaboration, ATLAS-CONF-2011-141.

[10] The CMS Collaboration, CMS-PAS-TOP-11-029.

[11] The ATLAS Collaboration, Submittes to JHEP (arXiv:1206.0257).

[12] The CMS Collaboration, CMS-PAS-TOP-11-028.

[13] The ATLAS Collaboration, Phys. Lett. B 712 (2012) 351-369

[14] The ATLAS Collaboration, Submittes to JHEP (arXiv:1205.2484).

[15] The CMS Collaboration, CMS-PAS-TOP-11-020.

[16] The ATLAS Collaboration, Submittes to EJPC (arXiv:1203.4211).

[17] The CMS Collaboration, CMS-PAS-TOP-11-030.

[18] The ATLAS Collaboration, ATLAS-CONF-2012-057.

[19] The ATLAS Collaboration, Phys. Rev. Lett. 108 (2012) 212001. 